\title{
Attention Cueing as a Means to Enhance Learning from an Animation
}

\author{
BJÖRN B. de KONING ${ }^{1 *}$, HUIB K. TABBERS ${ }^{1}$, REMY \\ M. J. P. RIKERS ${ }^{1}$ and FRED PAAS ${ }^{1,2}$ \\ ${ }^{1}$ Department of Psychology, Erasmus University Rotterdam, Rotterdam, The Netherlands \\ ${ }^{2}$ Educational Technology Expertise Center, Open University of The Netherlands, Heerlen, \\ The Netherlands
}

\begin{abstract}
SUMMARY
The question how animations should be designed so that learning is optimised, is still under discussion. Animations are often cognitively very demanding, resulting in decreased learning outcomes. In this study, we tried to prevent cognitive overload and foster learning by focusing the learners' attention to one element (i.e. process) of an animation using a cueing technique. Psychology students viewed an animation of the cardiovascular system and were subsequently given a comprehension test and a transfer test. One group studied the animation without a visual cue, while for another group a visual cue was added to the animation. Results indicated that cueing not only enhanced comprehension and transfer performance for cued information, but also for uncued information. It is concluded that cueing can be used as a technique to improve learning from an animation. Results are interpreted in terms of cognitive load theory (CLT). Copyright (C) 2007 John Wiley \& Sons, Ltd.
\end{abstract}

Dynamic visualisations, such as animations, showing complex dynamic processes, may be especially supportive in the construction of elaborated schemata. Both educational practitioners and instructional designers often assume that animations have important advantages over static graphics, especially in providing motion and trajectory information (Mayer \& Moreno, 2002; Rieber, 1990), making them a popular instructional tool. However, evidence is accumulating that animations are not instructionally superior to static graphics (Mayer, Hegarty, Mayer, \& Campbell, 2005) and most reviews have concluded that animations are at best no more and sometimes even less effective than the equivalent static graphics (Betrancourt \& Tversky, 2000; Hegarty, Kriz, \& Cate, 2003; Tversky, Morrison, \& Betrancourt, 2002). Despite the widespread use of animations in educational practice (Lowe, 2001), little is known about how such learning materials are cognitively processed (Lowe, 1999, 2004) and how they should be adapted to make learning more effective. Therefore, systematically studying the cognitive processes involved in learning from animations is of major importance.

Cognitive Load Theory (CLT; Sweller, 1988; Sweller, van Merriënboer, \& Paas, 1998) provides a theoretical framework that explains why learning from animations often fails. According to this theory, three sources of cognitive load can be imposed on learners when learning from animations. Intrinsic cognitive load is imposed by the information elements 
in the learning material and their interactions. Extraneous cognitive load is imposed on learners when mental activities, which are not directly related to learning (e.g. high visual search), are required to understand the material and is often the result of poorly designed instruction. Changing the instructional format can reduce extraneous load and promote learning. Germane cognitive load is imposed when information is presented in a way that learning is enhanced. For example, when mental activities such as elaboration and organisation of information result in the construction and automation of schemata. According to CLT, the combination of high intrinsic load (i.e. many interacting information elements) and high extraneous load (i.e. transience and high visual search) may account for suboptimal learning from animations.

The essential characteristic that distinguishes animations from static ways of presenting information is their higher complexity, as they incorporate motion and temporal aspects. Because information is transient, learners have to process current information and remember previous information simultaneously. If both current information and previous information have to be considered simultaneously to be understood, comprehension may fail because information presented during earlier phases of the animation may be forgotten or has become less clear. So, learning from an animation instead of a static picture may impose a higher cognitive load and thus may require other strategies, both cognitive and perceptual (Lowe, 1999).

Furthermore, a prerequisite for constructing coherent schemata of the subject matter domain displayed in an animation is that all relevant elements in the animation are attended to and extracted appropriately. However, animations are often visually too complex to be accurately understood, for example, because attention should be directed to several simultaneously occurring events (Hegarty et al., 2003; Schnotz, Böckheler, \& Grzondziel, 1999; Tversky et al., 2002). In a study by Lowe (1999) participants were presented with an animated training of how weather maps change in order to help them build a mental model of weather maps. They subsequently had to predict how the markings on a static weather map would change on the next day. Results revealed a perceptual dominance effect, implying that perceptually salient features of the animation drew learners' attention away from the thematically more relevant, but perceptually less salient features. This suggests that learning is enhanced when salient features correspond to thematically relevant aspects. Otherwise, the search for and extraction of relevant elements becomes a difficult additional task, which may impose high levels of extraneous load on learners relatively inexperienced in a domain who do not possess the necessary schemata to help them distinguish relevant from irrelevant information. In this case, an animation involving several simultaneously occurring events may never be effective for learning.

However, from a cognitive load perspective, animations may be less demanding and may become more effective when they are designed in a way that extraneous load is minimised. A possible way to reduce extraneous load, without reducing the informational richness of animations (e.g. motion and timing), is by focusing the learners' attention on relevant aspects in an animation by cueing them, which Betrancourt (2005) refers to as the attention-guiding principle. By adding a visual cue to a complex animation visual search should be reduced, thereby reducing extraneous load and allowing more cognitive resources to be allocated to learning.

\section{CUEING}

Cueing was originally defined as 'the addition of a non-content aspect of prose, which gives emphasis to certain aspects of the semantic content or points out aspects of the structure of 
the content' (Meyer, 1975, p.77). When applied to animations, cueing can be defined as the addition of non-content information that captures attention to those aspects that are important in an animation (e.g. coloring, arrows). So, cues are intended to guide the learners' cognitive processing but are not intended to provide new information (Mautone \& Mayer, 2001).

Although there is a large body of research showing the added value of cueing in texts (Loman \& Mayer, 1983; Lorch, 1989; Lorch \& Lorch, 1996; Lorch, Lorch, \& Inman, 1993), only a few studies have addressed the instructional value of cueing in other learning resources. Several studies, in which two separate sources of information, such as on-screen or narrated text and pictorial information have to be integrated, clearly demonstrate that cueing can be very effectively used to reduce visual search in a multimedia explanation (Jeung, Chandler, \& Sweller, 1997; Kalyuga, Chandler, \& Sweller, 1999; Tabbers, Martens, \& van Merriënboer, 2004). Kalyuga et al. (1999), for example, found that the use of color coding as a visual cue to establish a link between textual and pictorial information, resulted in much better learning outcomes. However, mixed results have been obtained with respect to the effect of cueing on cognitive load, indicating that sometimes cognitive load is slightly lowered by adding visual cues (Kalyuga et al., 1999), while in other situations cueing has no significant effect on reported mental effort (Tabbers et al., 2004). However, this does not necessarily imply that cueing was not effective in reducing extraneous load. In general, instructions should be designed to decrease extraneous load and optimise germane load. Although the overall amount of cognitive load may not differ, the relative contribution of extraneous and germane load can vary with different instructions.

The only study that directly addressed cueing in an animation showed that the incorporation of visual cues to an animation did not enhance learning (Mautone \& Mayer, 2001). In this study, a narrated animation was studied in which the narration and the animation could either be cued or uncued. No significant effects of visual cueing were found for the animation on retention and transfer. To explain these results, Mautone and Mayer argued that the animation was too simple and contained few distracting elements. In other words, the animation represented no simultaneously occurring processes, so cues were unnecessary in directing attention and reducing extraneous load.

Cueing has only been investigated in a multimedia context to reduce the search for appropriate referents in a text and accompanying picture. Yet, little is known about how cueing affects learning from visual-only instructions in animations. In addition, cueing in animations has only been studied in a low visual search situation. As cueing is expected to be most effective in high visual search situations, valid conclusions about cueing in animations can only be made when investigated under high visual search conditions.

The aim of the present study was to determine whether cueing a complex animation of the cardiovascular system would result in better learning outcomes (i.e. comprehension and transfer) than presenting the same animation without a visual cue. It was hypothesised that the animation would only enhance learning for learners with moderate prior knowledge when irrelevant visual search was reduced by guiding attention by adding a visual cue. To avoid ordering and segmentation effects as a result of cueing different processes serially, only one process was visually cued. Based on CLT it could be predicted that adding a visual cue to a visually complex animation will accomplish a decrease in extraneous load and hence makes the instructional animation more effective in terms of learning (i.e. germane load). According to CLT, experiencing less extraneous load during learning frees up working memory resources (Sweller, 1999). Therefore, in line with Kalyuga et al. (1999), a 
small effect of cueing on either mental effort scores or test performance is predicted. That is, there may be equal test performance but less mental effort involved in studying the animation with an added visual cue than when no visual cue is present in the animation. Alternatively, both conditions may experience the same amount of mental effort but yield better performance when a visual cue is added to the animation. To address not only the effectiveness of instructions by looking at test results but also the efficiency of the instructions, a subjective measure of mental effort was also administered after the comprehension and transfer test to assess whether the same performance took the same amount of mental effort. Furthermore, by cueing one complete process, attention is directed to a meaningful unit of information, that is, a functional important part of the cardiovascular system. Therefore, it is reasonable to assume that cueing has a large effect on the performance of the questions about the cued process. To investigate this possibility, a more specific prediction was that cueing one process would result in better comprehension and transfer outcomes on the questions concerning that process.

\section{METHOD}

\section{Participants}

The participants were 40 undergraduate psychology students (10 males and 30 females) from the Erasmus University Rotterdam. Age ranged between 19 and 33 years. All were native Dutch speakers and received partial course credit or a small monetary reward for their participation. Participants had normal or corrected to normal vision, were unaware as to the exact purpose of the study, and gave informed consent.

\section{Design}

The experiment conformed to a 2 Cueing (Yes vs. No) $\times 2$ Question-type (Valves vs. Other processes) mixed factorial design. The factor Question-type was manipulated within participants and the factor Cueing was manipulated between participants resulting in two conditions: the No-Cueing (NC) condition and the Cued-Animation (CA) condition. Participants were randomly assigned to the experimental conditions, with 20 participants in the NC-condition and 20 participants in the CA-condition. In the NC-condition, participants were presented an animation of the cardiovascular system without any cues. Participants in the CA-condition viewed the same animation, but with the process of how the valves of the heart work cued. To determine whether both groups differed in their amount of prior knowledge of the cardiovascular system, we investigated their prior knowledge by adding up the scores on a checklist (see Appendix A) and using the students' self-rating concerning their knowledge about the cardiovascular system. Analysis of this prior knowledge score indicated no significant differences between the cued animation condition $(M=6.9, S D=3.19)$ and the uncued animation condition $(M=5.4, S D=3.00)$, $t(38)=1.686, p>0.05$.

\section{Materials}

The materials consisted of an animation, a participant questionnaire, a cognitive load rating scale, a comprehension test, and a transfer test. All materials were combined into one 
computer application and this programme was presented on a 17" LCD color computer screen.

An animation of the cardiovascular system (Figure 1) was created for the purpose of this study. To assure that the information presented in the animation was a realistic depiction of the cardiovascular system, the animation was developed in collaboration with two physicians. The animation consisted of five basic processes of the cardiovascular system that are distinguished in medical education: the circulatory system, the electrical system, the pulmonary circulation, the systemic circulation, and the valves system. All five processes have their own role and function at their own pace. However, all processes interact with each other and hence each process is contingent on the proper functioning of the other processes. In short, the animation shows how the heart expands as it fills with blood and shrinks as blood is pumped out of the heart, how the lungs expand when oxygen flows into the lungs to the alveoli and shrink when waste products flow from the alveoli out of the lungs, how and when the valves of the heart open and close, how and when the electrical system is activated and how this activation spreads along the heart muscle causing it to contract, the direction and pathway of the blood flow, where and when oxygen is taken up in the blood and waste products are given off, where and when oxygen is given off to muscles in the body and waste products are taken up, the timing between all these simultaneously occurring processes. Our main goal was to enhance knowledge of the dynamics of the cardiovascular system rather than learning the specific names and locations for each part of the cardiovascular system. Therefore, we did not include information about terminology or anatomy of the cardiovascular system. Moreover, participants had taken at least some basic biology courses in high school that included the cardiovascular system and its basic components. Prior testing indicated that the names and

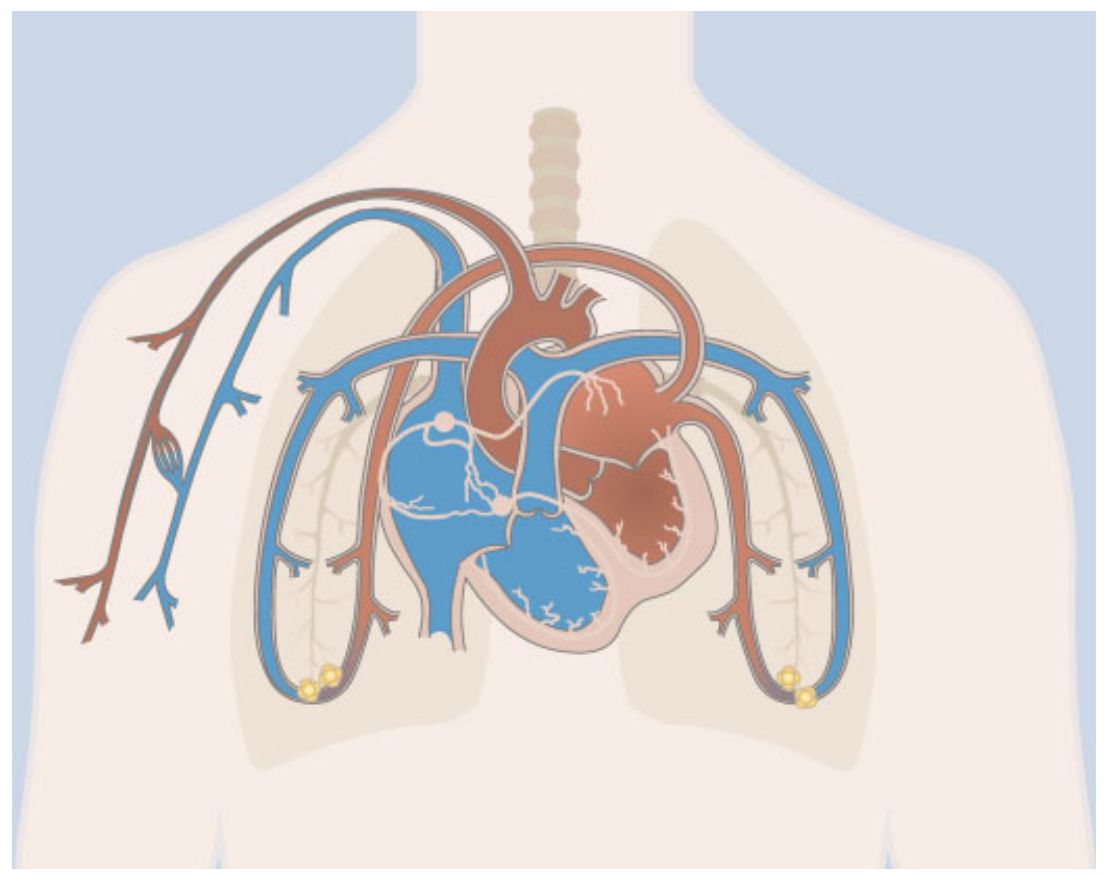

Figure 1. Screenshot of the animation of the cardiovascular system 
locations of these components, but not their functions, were well known and that a total of six cardiac cycles was the average number of cycles to reach quite a good understanding of the information presented in the animation. Therefore, a total of six cardiac cycles was shown in the animation, each lasting for 10 seconds. The total duration of the animation was 60 seconds and the animation did not contain any textual information. So, participants had to rely only on the visual information to extract meaning from the animation.

Two versions of the same animation of the cardiovascular system were used in this study. One version showed the workings of the cardiovascular system without a visual cue and one version showed the workings of the cardiovascular system but now the valves of the heart were visually cued. A complete functionally important process was cued instead of specific subcomponents of such a process, because a study by Lowe (1999) suggests that cueing small elements diverges attention away from the more relevant elements, which imposes a high extraneous load and hinders the construction of elaborated schemata. The valves system was chosen as the process to be cued, because this system is an important functional part of the cardiovascular system, but is not very salient (Lowe, 1999). To ensure the visual cue attracted attention appropriately and was not conceived of as part of the animation, the visual cue appeared 10 seconds after the start of the animation, instead of right from the beginning and was visible until the end of the animation. Thus, the valves were cued after one cardiac cycle was completed and remained highlighted during five cardiac cycles. Cueing was done by slightly darkening all elements in the animation except the valves of the heart, which can be thought of as a spotlight-effect (Figure 2), that was defined in this study as a situation in which one process is highlighted and, therefore, stands out against all other processes in the animation. It is important to note, that all uncued elements could still be accurately perceived. So, with the exception of the cued process, both versions were identical. Both versions were created using Macromedia Flash 7.0 (Macromedia, 2004). Furthermore, to ensure that any effects of cueing could not be confounded with interactivity, which introduces an additional way, next to cueing, to manage the perceptual complexity by controlling the pace and direction of the animation (Betrancourt, 2005), it was impossible to stop or to replay the animation, which is comparable to prior research (Mautone \& Mayer, 2001; Mayer, Heiser, \& Lonn, 2001).

The participant questionnaire, based on Mayer and Moreno (1998), asked participants to indicate their gender, age, years of university education, and knowledge of the cardiovascular system. The knowledge of the cardiovascular system was assessed using a 1-item self-rating and a 4-item checklist (see Appendix A). On the 1-item self-rating question participants were asked to indicate their knowledge of the heart and the blood flow on a 5-point scale ranging from very little (1) to very much (5). The 4-item checklist consisted of items concerning knowledge about the cardiovascular system. For example, participants were asked whether they had followed any biology classes during their secondary education or whether they knew someone in their inner circle with a heart condition. All questions regarding prior knowledge were added up to get a score, indicating overall experience of the cardiovascular system, ranging from no experience (0), to high experience (14).

The mental effort measure used in this study was a 9-point rating scale, ranging from very, very easy (1), to very, very difficult (9), and was developed by Paas (1992). Subjective self-ratings of invested mental effort were chosen, because they are non-intrusive and they give a reliable and valid indication of experienced complexity of the task at hand (Paas, Tuovinen, Tabbers, \& Van Gerven, 2003; Paas \& van Merriënboer, 1993, 1994; Paas, van Merriënboer, \& Adam, 1994). 


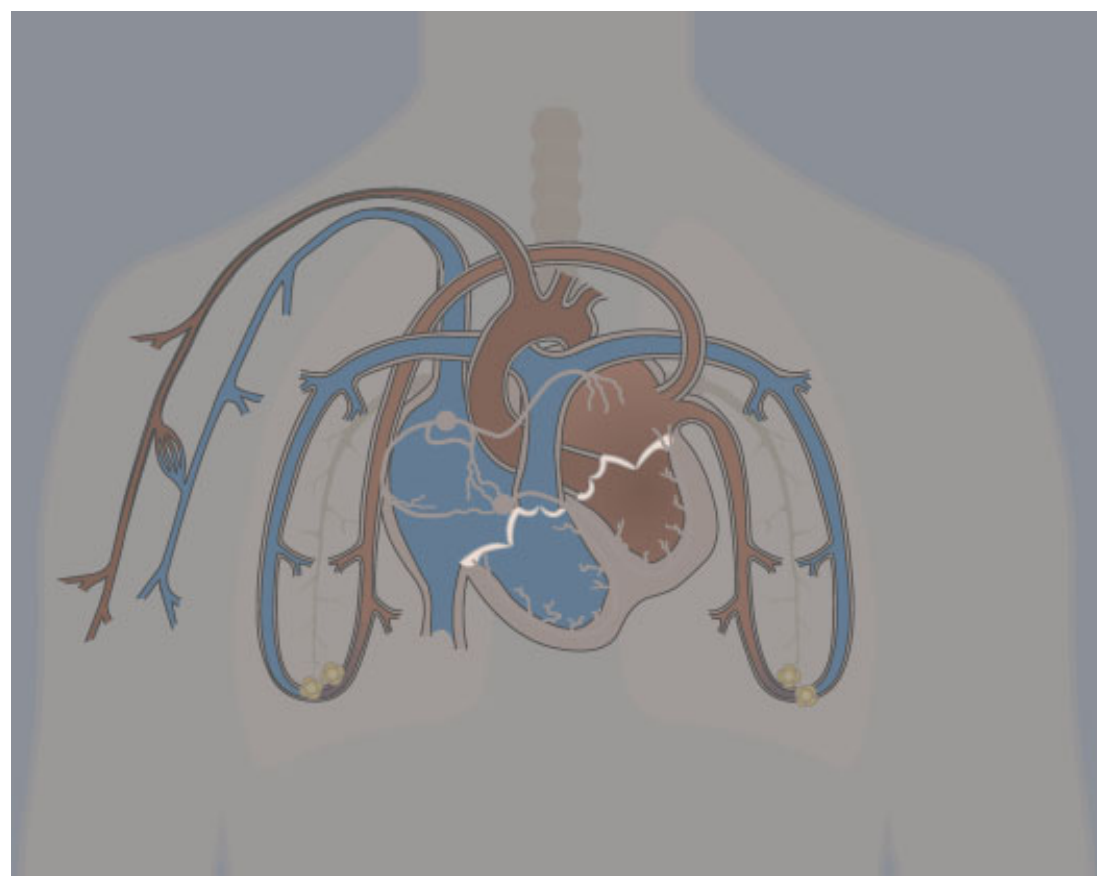

Figure 2. Screenshot of the animation of the cardiovascular system with the valves of the heart visually cued

The comprehension test consisted of 38 multiple-choice questions, asking participants to indicate the correct answer out of four options. The multiple-choice questions covered all five processes and elements included in the animation. The multiple choice questions could be divided into 10 questions directly related to the functioning of the valves of the heart and 28 questions about the other four processes of the cardiovascular system (e.g. the electrical system). To assure that the comprehension test included questions about structural, procedural, and temporal aspects of the processes of the cardiovascular system, we used these three categories as an aid to develop the comprehension questions. Whenever possible, questions were formulated visually and hence made minimal use of terminology. Appendix B shows examples of comprehension questions for each category and process. All comprehension questions were evaluated by a physician.

The transfer test consisted of 10 open-ended questions and covered all processes and elements included in the animation. Examples of transfer questions are: 'Explain why the heart contracts faster when someone holds his breath?', 'Explain what causes the valves of the heart to open and close', 'Which part of the blood flow contains hardly any waste products? Explain your answer', 'The workings of the electrical system can be divided into two steps. Explain why this is'. These questions can only be answered byámaking inferences from the information presented in the animation and, therefore, provides a good measure of the quality of the schema that is constructed (Mayer, 2001). Out of all 10 transfer questions, two explicitly asked to make inferences about the functioning of the valves of the heart. All transfer questions were evaluated by a physician. 


\section{Procedure}

Participants were tested in groups up to four people. They were seated at a computer screen and worked through the entire application at their own pace. First of all each participant was asked to fill out the participant questionnaire. Then they read instructions that stated that the animation should be attended to carefully, and they were explicitly encouraged to comprehend how the cardiovascular system works. Furthermore, it was announced that they would be tested afterwards on the cardiovascular system. Depending on the condition, they viewed either the cued animation or the uncued animation. After viewing the animation, participants were given a mental effort rating scale on which they could rate their invested mental effort by clicking on one of the options of the rating scale. Subsequently, participants received the comprehension test. Each question was presented individually on the computer screen in a random order. Participants could answer the multiple-choice questions by clicking on the answer they believed to be correct. After finishing each question, participants could advance to the next question by clicking a 'next-button'. Participants could not go back to questions they had already answered. Subsequently, a transfer test was given. As with the comprehension questions, each question was presented individually on the computer screen in a random order. Participants could answer the transfer questions by typing their answer in an on-screen textbox by using the keyboard. There was no time limit for the tests and each test was followed by a mental effort rating. The total procedure lasted for about 45 minutes.

\section{Analysis}

The comprehension questions were scored by adding up the correct responses for each question. Each right answer yielded 1 point. Separate counts were made for questions concerning the cued process (i.e. the valves of the heart) and the questions concerning all other processes. This resulted in two comprehension scores: a comprehension score (between 0 and 10) for questions about the cued process and a comprehension score (between 0 and 28) for all other comprehension questions. Cronbach's alpha for the overall comprehension test was 0.70 , which can be considered an acceptable degree of internal consistency in this study given that $a$ priori we did not expect the internal consistency to be very high because the animation lasted only 60 seconds and involved many simultaneously occurring processes, so it was assumed that participants would differ in the information that was extracted.

For the transfer test, we developed a scoring form with 48 core idea units distributed over all questions. Scoring was done by counting for each question how many of the core idea units per question were included in the participant's responses. As with the comprehension test, separate counts were made for questions concerning the cued process (i.e. the valves of the heart) and the questions concerning all other processes. This resulted in two transfer scores: a transfer score (between 0 and 11) for questions about the cued process and a transfer score (between 0 and 37 ) for all other transfer questions. Cronbach's alpha for the overall transfer test was 0.84 .

A scorer who was unaware of treatment condition, scored all responses on the comprehension test and the transfer test, for each participant. A randomly selected subset of $25 \%$ of the tests was scored by a second scorer. Agreement between both scorers was $100 \%$ on the comprehension test, and $97 \%$ on the transfer test. Based on these results, it was concluded that the scoring procedure was sufficiently reliable and the scores from the first scorer could be used. 


\section{RESULTS}

The dependent variables were comprehension score for questions about the cued process, comprehension score for all other comprehension questions, transfer score for questions about the cued process, a transfer score for all other transfer questions, and mental effort score during the animation and the mental effort during the comprehension test and the transfer test. The scores on the two comprehension measures, the two transfer measures, and the three mental effort measures were subjected to separate multivariate analysis of variance (MANOVA) with Cueing (Yes vs. No) as the between-participants factor. Additionally, separate univariate analysis of variance (ANOVA) was conducted. All statistical tests were carried out using a 0.05 significance level. Effect sizes were calculated using Cohen's measure of effect size (indicated by $d$ ). According to the conventions defined by Cohen (1988) $d$ values of $0.2,0.5$, and 0.8 correspond to small, medium, and large effect sizes respectively.

\section{Outcome measures}

The MANOVA on the comprehension scores reveals a significant effect of cueing, Wilks' $\lambda=0.85, F(2,37)=3.29, p<0.05$. The results shown in Table 1 , which provides the means and standard deviations of the univariate analyses on comprehension and transfer for the two conditions, indicate that participants in the CA-group performed better than the participants in the NC-group with respect to the comprehension questions about the valves, $F(1,38)=5.21, M S E=1.39, p<0.05, d=0.72$. Interestingly, results on the comprehension questions about the other processes also indicate an advantage for the CA-group as compared to the NC-group, $F(1,38)=4.29, M S E=16.39, p<0.05, d=0.65$.

In addition, the MANOVA on the transfer scores also indicates a significant effect of cueing, Wilks' $\lambda=0.84, F(2,37)=3.66, p<0.05$. As can be seen in the univariate outcomes shown in Table 1, a cueing effect was found for the transfer questions concerning the valves, with the CA-group scoring significantly better than the NC-group, $F(1$, $38)=5.28, M S E=3.45, p<0.05, d=0.73$. Participants who received the cued animation not only obtained higher learning outcomes on the questions concerned with the valves system than the participants who received an uncued animation, but also on transfer questions concerning the other processes of the cardiovascular system, $F(1,38)=7.09$, $M S E=18.27, p<.05, d=0.84$, which indicates a strong effect of cueing on uncued processes of the animation.

Table 1. Mean scores (and standard deviations) on the comprehension test and the transfer test for the CA-group and the NC-group

\begin{tabular}{lccccc}
\hline & \multicolumn{2}{c}{ CA-group } & & \multicolumn{2}{c}{ NC-group } \\
\cline { 2 - 3 } & Number Correct & $S D$ & & Number Correct & $S D$ \\
\hline Comprehension & & & & & \\
$\quad$ Valves (0-10) & 6.7 & 1.3 & & 5.9 & 1.0 \\
$\quad$ Other processes (0-28) & 15.5 & 3.7 & & 12.9 & 4.3 \\
Transfer & & & & 1.7 & 1.5 \\
$\quad$ Valves (0-11) & 3.1 & 2.1 & & 6.8 & 4.0 \\
$\quad$ Other processes $(0-37)$ & 10.4 & 4.6 & & \\
\hline
\end{tabular}


To determine the cognitive load of the different tasks imposed on the learner, mental effort was measured after studying the animation and after each test. The MANOVA on the mental effort measures reveals no significant effect of cueing, Wilks' $\lambda=0.96, F(3$, $36)=0.53, p>0.05$. In the first row of Table 2, which displays the means and standard deviations of the univariate analyses for the mental effort scores after completing each task for the two conditions, it can be seen that mental effort while studying the animation did not significantly differ between the CA-group and the NC-group, $F(1,38)=0.17, M S E=2.36$, $p>0.05$. When looking at the mental effort measures after the comprehension and transfer test, which are displayed in the second and third row of Table 2, results reveal that none of these measures significantly differed between the two conditions, $F(1,38)=0.99$, $M S E=2.05, p>0.05$ and $F(1,38)=0.04, M S E=2.78, p>0.05$, respectively.

\section{DISCUSSION}

This study investigated whether studying a complex animation with a visual cue would result in better comprehension and transfer performance than when the animation was studied without a visual cue. The results confirmed the hypothesis, indicating that cueing can be successfully used as a technique to enhance performance when learning from animations without significant differences between conditions in mental effort. By guiding attention to the relevant aspects in the animation, working memory resources can be allocated to learning more efficiently. Also, the results on the transfer test suggest that cueing assisted learners in forming more coherent schemata, than when the animation is not cued. Thus, learners benefited from cueing and were able to process the presented information satisfactorily because cueing provided attentional guidance that may have reduced visual search. However, to support this claim additional research is needed, for example by varying visual search complexity of the animation or by applying an eye-tracking methodology to see how cueing affects overt attention allocation. These findings extend prior research on cueing suggesting that visual cueing not only works when applied to texts (Lorch, 1989; Lorch et al., 1993), or the combination of pictorial and textual information (Kalyuga et al., 1999) but also when applied to animations that do not involve any narrations. The question whether cueing also enhances learning in animations that contain narrations remains to be addressed.

Contrary to our expectations, we did not find a decrease in mental effort while studying the animation when focusing attention in that animation, which is consistent with results obtained by Tabbers and colleagues (2004). In the present study, the mental effort scores were fairly low. This could be an indication that the animation was not complex enough or that people invested too little effort. However, low or average mental effort scores are commonly reported (Mayer \& Chandler, 2001). Given that the scores on the

Table 2. Mean mental effort scores (and standard deviations) for the CA-group and the NC-group

\begin{tabular}{|c|c|c|c|c|}
\hline & \multicolumn{2}{|c|}{ CA-group } & \multicolumn{2}{|c|}{ NC-group } \\
\hline & $M$ & $S D$ & $M$ & $S D$ \\
\hline Mental effort animation (1-9) & 4.4 & 1.5 & 4.6 & 1.6 \\
\hline Mental effort comprehension (1-9) & 6.3 & 1.4 & 6.7 & 1.5 \\
\hline Mental effort transfer (1-9) & 6.1 & 1.4 & 6.2 & 1.9 \\
\hline
\end{tabular}


comprehension and transfer test are not very high and that the present animation included several simultaneously occurring events and, hence, consisted of a reasonable degree of complexity, this first explanation does not seem very likely. Alternatively, our latter explanation seems a more plausible explanation as it is in line with the idea that motivational factors may play a crucial role in learning by mediating the amount of cognitive processing (Moreno, 2006).

Interestingly, results revealed that cueing not only improved comprehension and transfer for the cued process, but also enhances knowledge of the uncued processes. These results go beyond our hypothesis that cueing one process within an animation leads to better comprehension of that process than when that process is not visually highlighted. Despite the fact that the mechanisms behind cueing in animations are far from well known, several interpretations can be offered to explain this result. For example, understanding the workings of the valves system might make other processes easier, by way of functionality. That is, the valves system is a special process in the sense that it is located in the middle of the heart and serves a central function in the cardiovascular system, for example, in regulating the direction and trajectory of blood flowing in and out off each chamber of the heart. So, if the valves are understood, other processes might become easier to understand as well (i.e. the direction of the blood flow). However, we use CLT to interpret the present findings and contend that, when studying a cued animation more cognitive resources can be allocated to germane load instead of extraneous load, that is, processes relevant for learning. At a perceptual level, the visual cue divides the animation in a cued segment and an uncued segment. At a cognitive level, this may have consequences on the allocation of attention, and hence, the distribution of working memory resources. That is, as each segment can be processed in isolation, less working memory capacity is used, which in turn can be used for the construction and integration of elaborated schemata. This seems a plausible explanation, which also acknowledges the finding that cueing also enhances learning outcomes for the uncued elements. Further research is needed to test this account, for example by shortening the time to study the animation to see if mental effort is reduced when no time is given to study uncued processes and integrate cued and uncued elements.

Our results and interpretations are limited by the learners, the nature of the materials, and the learning situation. First, the learners had at least some basic understanding of the cardiovascular system and hence can be characterised as having average background knowledge. Based on the expertise reversal effect (Kalyuga, Ayres, Chandler, \& Sweller, 2003 ) it would be reasonable to assume that cueing has differential effects on more and less knowledgeable learners. Second, the animation was short, involved no narrations, involved only one cued process and the speed of the animation was not chosen on theoretical grounds but it was based on the standard speed of the software package with which the animation was developed. It is not clear whether the present results would also apply to animations that are longer, involve narrations, contain more than one cued process, and are played at different speeds. For example, it could be argued that playing the animation at different speeds differentially affects the effectiveness of cueing, with fast animations urging the need for a visual cue to keep track of all changes while in a slow animation a cue is unnecessary in guiding attention because all elements can be attended to appropriately. More research is needed to further investigate these issues as potential moderating factors. Third, the animation was shown just once and lacked the opportunity to interact, which seems a rather artificial situation. In general, animations in educational practice involve some kind of interactivity (e.g. stop, and replay) and can be viewed multiple times. It remains to be addressed whether the same results are obtained when these issues are taken 
into account. Overall, these limitations put some constraints on the generalisability of our findings. Future research is needed to determine whether visual cueing differentially affects learners (such as learners with different levels of expertise), different kinds of learning materials (such as animations with different speeds), and other learning situations (such as animations incorporating interactivity).

From a practical point of view, this study opens a new view on learning from animations and the design of multimedia instructions. The cueing-effect obtained in this study has a direct implication for the design of animations. Learners often are unable to process the presented information in an animation and have difficulties extracting relevant information from it. While it is possible to use a simplified static picture instead of an animation to promote comprehension (Butcher, 2006), valuable information such as timing aspects and dynamics in an animation are lost when reducing complexity. Based on the present study we provide a new way of reducing the visual complexity of animations in order to promote learning in the form of cueing. Cueing can play a crucial role in the comprehension of an animation by a more efficient use of working memory resources. Moreover, cueing may have the additional advantage of organising information presented in the animation. In conclusion, incorporating a visual cue in the design of an animation seems an interesting tool for instructional designers to enhance learning from an animation.

\section{ACKNOWLEDGEMENTS}

The authors would like to thank Eveline Osseweijer for her assistance in developing the animation of the cardiovascular system.

\section{REFERENCES}

Betrancourt, M. (2005). The animation and interactivity principles in multimedia learning. In R. E. Mayer (Ed.), Cambridge handbook of multimedia learning (pp. 287-296). New York: Cambridge University Press.

Betrancourt, M., \& Tversky, B. (2000). Effect of computer animation on users' performance: A review. Le Travail Humain, 63, 311-329.

Butcher, K. R. (2006). Learning from text with diagrams: Promoting mental model development and inference generation. Journal of Educational Psychology, 98, 182-197.

Cohen, J. (1988). Statistical power analysis for the behavioral sciences (2nd ed.). Hillsdale, NJ: Erlbaum.

Hegarty, M., Kriz, S., \& Cate, C. (2003). The roles of mental animations and external animations in understanding mechanical systems. Cognition and Instruction, 21, 325-360.

Jeung, H., Chandler, P., \& Sweller, J. (1997). The role of visual indicators in dual sensory mode instruction. Educational Psychology, 17, 329-343.

Kalyuga, S., Ayres, P., Chandler, P., \& Sweller, J. (2003). The expertise reversal effect. Educational Psychologist, 38, 23-31.

Kalyuga, S., Chandler, P., \& Sweller, J. (1999). Managing split-attention and redundancy in multimedia instruction. Applied Cognitive Psychology, 13, 351-371.

Loman, N. L., \& Mayer, R. E. (1983). Signaling techniques that increase the understandability of expository prose. Journal of Educational Psychology, 75, 402-412.

Lorch, R. F. (1989). Text signaling devices and their effects on reading and memory processes. Educational Psychology Review, 1, 209-234.

Lorch, R. F., \& Lorch, E. P. (1996). Effects of organizational signals on free recall of expository text. Journal of Educational Psychology, 88, 38-48.

Lorch, R. F., Lorch, E. P., \& Inman, W. E. (1993). Effects of signaling topic structure on text recall. Journal of Educational Psychology, 85, 281-290. 
Lowe, R. K. (1999). Extracting information from an animation during complex visual learning. European Journal of Psychology of Education, 14, 225-244.

Lowe, R. K. (2001). Understanding information presented by complex animated diagrams. In J.-F. Rouet, J. Levonen, \& A. Biardeau (Eds.), Multimedia learning-Cognitive and instructional issues (pp. 65-74). Amsterdam: Elsevier Science Ltd.

Lowe, R. K. (2004). Interrogation of a dynamic visualization during learning. Learning and Instruction, 14, 257-274.

Macromedia. (2004). Flash MX 7.0 [Computer software]. San Francisco: Author.

Mautone, P. D., \& Mayer, R. E. (2001). Signaling as a cognitive guide in multimedia learning. Journal of Educational Psychology, 93, 377-389.

Mayer, R. E. (2001). Multimedia learning. New York: Cambridge University Press.

Mayer, R. E., Hegarty, M., Mayer, S., \& Campbell, J. (2005). When static media promote active learning: Annotated illustrations versus narrated animations in multimedia instruction. Journal of Experimental Psychology: Applied, 11, 256-265.

Mayer, R. E., Heiser, J., \& Lonn, S. (2001). Cognitive constraints on multimedia learning: When presenting more material results in less understanding. Journal of Educational Psychology, 93, $187-198$.

Mayer, R. E., \& Moreno, R. (1998). A split-attention effect in multimedia learning: Evidence for dual processing systems in working memory. Journal of Educational Psychology, 90, 312-320.

Mayer, R. E., \& Moreno, R. (2002). Animation as an aid to multimedia learning. Educational Psychology Review, 14, 87-99.

Meyer, B. J. F. (1975). The organization of prose and its effects on memory. New York: Elsevier.

Moreno, R. (2006). Does the modality principle hold for different media? A test of the methodaffects-learning hypothesis. Journal of Computer Assisted Learning, 22, 149-158.

Paas, F. (1992). Training strategies for attaining transfer of problem-solving skill in statistics: A cognitive-load approach. Journal of Educational Psychology, 84, 429-434.

Paas, F., Tuovinen, J. E., Tabbers, H., \& Van Gerven, P. W. M. (2003). Cognitive load measurement as a means to advance cognitive load theory. Educational Psychologist, 38, 63-71.

Paas, F., \& van Merriënboer, J. J. G. (1993). The efficiency of instructional conditions: An approach to combine mental-effort and performance measures. Human Factors, 35, 737-743.

Paas, F., \& van Merriënboer, J. J. G. (1994). Variability of worked examples and transfer of geometrical problem-solving skills: A cognitive-load approach. Journal of Educational Psychology, 86, 122-133.

Paas, F., van Merriënboer, J. J. G., \& Adam, J. J. (1994). Measurement of cognitive load in instructional research. Perceptual and Motor Skills, 79, 419-430.

Rieber, L. P. (1990). Using computer animated graphics with science instruction with children. Journal of Educational Psychology, 82, 135-140.

Schnotz, W., Böckheler, J., \& Grzondziel, H. (1999). Individual and cooperative learning with interactive animated pictures. European Journal of Psychology and Education, 14, 245-265.

Sweller, J. (1988). Cognitive load during problem solving: Effects on learning. Cognitive Science, 12, 257-285.

Sweller, J. (1999). Instructional design in technical areas. Camberwell, Australia: Australian Council for Educational Research.

Sweller, J., van Merriënboer, J. J. G., \& Paas, F. (1998). Cognitive architecture and instructional design. Educational Psychology Review, 10, 251-296.

Tabbers, H. K., Martens, R. L., \& van Merriënboer, J. J. G. (2004). Multimedia instructions and cognitive load theory: Effects of modality and cueing. British Journal of Educational Psychology, $74,71-81$.

Tversky, B., Morrison, J. B., \& Betrancourt, M. (2002). Animation: Can it facilitate? International Journal of Human-Computer Studies, 57, 247-262. 


\section{APPENDIX A}

\section{CHECKLIST OF PRIOR KNOWLEDGE OF THE CARDIOVASCULAR SYSTEM}

\section{1-Item self-rating}

How much knowledge do you have about the cardiovascular system?

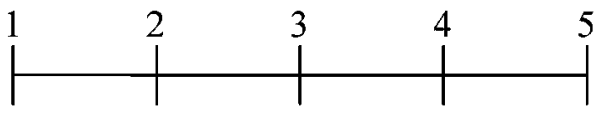

very little little not little, much very much

not much

\section{4-Item checklist}

Did you take biology classes in your final exam in high school?
$\square$ Yes
$\square$ No

In high school I took biology classes on the following level:

$\square$ I did not take biology classes

$\square$ Biology 1

Biology 2

Biology 1 and 2

I have taken biology classes after high school.

$\square$ Yes

I know someone from my inner circle who has suffered from a heart condition or has had a treatment to prevent it during the last 5 years.

$\square$ Yes

No

\section{APPENDIX B}

\section{EXAMPLES OF COMPREHENSION QUESTIONS FOR EACH CATEGORY}

(1) Valves system

When this valve, as indicated on the picture is open, then ...

(a) Blood flows from the undermost compartment to the uppermost compartment of the heart

(b) Blood flows to the lungs

(c) Blood flows from the uppermost compartment to the undermost compartment of the heart

(d) Blood flows into the body

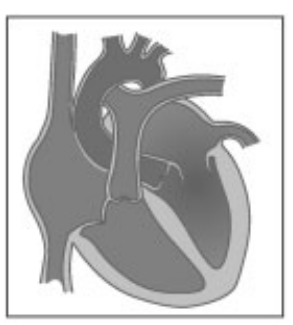


(2) Electrical system

Which picture shows the course of the electrical system correctly?

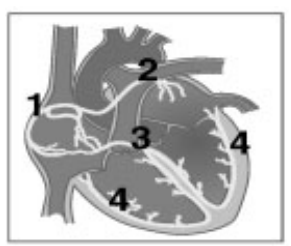

(a)

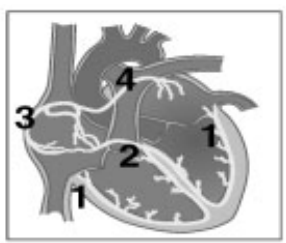

(b)

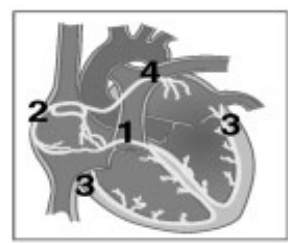

(c)

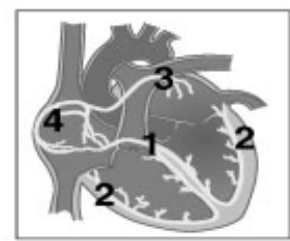

(d)

(3) Pulmonary circulation

Blood enters the heart from the body. How many times does the heart contract before blood enters the lungs?
(a) 1
(b) 2
(c) 3
(d) 4

(4) Circulatory system

Do the pulmonary alveoli (see picture) change colors?

(a) Yes, with every contraction of the heart

(b) Yes, with the storage and release of oxygen

(c) No, the pulmonary alveoli only get bigger

(d) No, the pulmonary alveoli stay the same

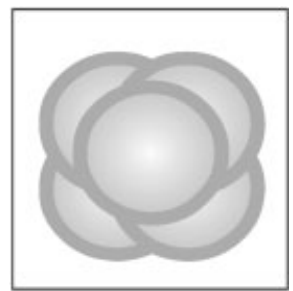

(5) Systemic circulation

How does blood travel from the left side of the heart to the right side of the heart?

(a) Blood can not go from one side of the heart to the other

(b) Through the body

(c) Through the lungs

(d) Through the body and the lungs

(6) Structure

How many compartments are there in the heart?
(a) 1
(b) 2
(c) 3
(d) 4

(7) Procedure

Within the heart blood flows from one compartment to the other. Which picture shows the direction of the blood flow correctly?

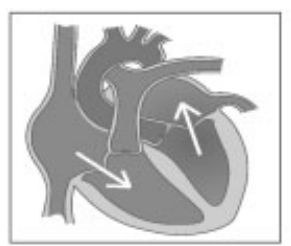

(a)

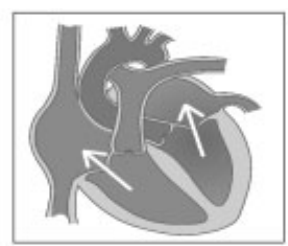

(b)

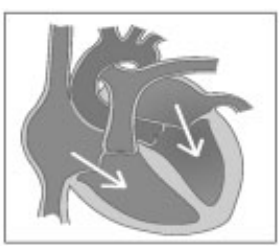

(c)

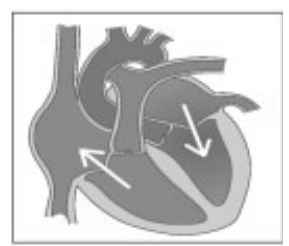

(d) 


\section{(8) Timing}

The exchange of oxygen in the body occurs ... the exchange of oxygen in the lungs?
(a) Before
(b) After
(c) Simultaneously with
(d) None of the above answers is correct 